\title{
Conditionally replicative adenovirus carrying shRNA targeting EZH2 inhibits prostate cancer growth and invasion
}

\author{
SHI-GAO XU ${ }^{1 *}$, JUN-JIE YU $^{1 *}$, QUN SHI $^{1}$, QUAN NIU $^{2}$, ZHE GUO $^{2}$, BAO-YU GUO $^{2}$, \\ GUANG-CHEN ZHOU ${ }^{1}$, XIAO GU ${ }^{1}$ and YIN-XIA WU ${ }^{3}$ \\ ${ }^{1}$ Department of Urology, Clinical Medical College of Yangzhou University, Subei People's Hospital of Jiangsu Province, \\ Yangzhou, Jiangsu 225001; ${ }^{2}$ Department of Urology, Dalian Medical University, Dalian, Liaoning 116044; \\ ${ }^{3}$ Department of Oncology, Clinical Medical College of Yangzhou University, Subei People's Hospital of Jiangsu Province, \\ Yangzhou, Jiangsu 225001, P.R. China
}

Received July 15, 2018; Accepted April 24, 2019

DOI: 10.3892/or.2019.7157

\begin{abstract}
The present study aimed to construct conditionally replicative adenovirus (CRAds) carrying small hairpin (sh)RNA targeting enhancer of zeste homolog 2 (EZH2), in order to study its effect on inhibiting prostate cancer (PCa) cell growth and invasion. Immunohistochemical analyses of EZH2 was performed in tumor tissue samples from PCa and benign prostate hyperplasia (BPH). The human telomerase reverse transcriptase (hTERT) promoter was chosen to transcriptionally control EZH2 gene expression to obtain adenoviral replication (Ad-hTERT-EZH2shRNA) in human PCa cell lines. The inhibitory effect of Ad-hTERT-EZH2shRNA on EZH2 expression was evaluated by reverse transcription-quantitative polymerase chain reaction and western blot analyses. Cell Counting Kit-8 assays were used to examine the effects of the Ad-hTERT-EZH2shRNA on cell proliferation. Transwell Matrigel invasion assays were used to detected cell invasion. Immunohistochemistry showed that EZH2 staining was stronger in castration-resistant prostate cancer (CRPC) samples, compared with androgen-dependent prostate cancer (ADPC) samples, and was absent in BPH. Furthermore, EZH2 expression knockdown suppressed $\mathrm{PCa}$ cell proliferation and invasion.
\end{abstract}

Correspondence to: $\mathrm{Dr}$ Xiao Gu, Department of Urology, Clinical Medical College of Yangzhou University, Subei People's Hospital of Jiangsu Province, 98 Nan Tong West Road, Yangzhou, Jiangsu 225001, P.R. China

E-mail: xgu@yzu.edu.cn

Dr Yin-Xia Wu, Department of Oncology, Clinical Medical College of Yangzhou University, Subei People's Hospital of Jiangsu Province, 98 Nan Tong West Road, Yangzhou, Jiangsu 225001, P.R. China

E-mail: jacy516@163.com

*Contributed equally

Key words: enhancer of zeste homolog 2, conditionally replicative adenovirus, human telomerase reverse transcriptase, prostate cancer, castration-resistant prostate cancer, treatment
In addition, it was found that Ad-hTERT-EZH2shRNA selectively replicated and significantly reduced the expression of EZH2 in PCa cells lines. The growth ability and invasion of DU145 and PC3 cells in vitro was effectively inhibited by Ad-hTERT-EZH2shRNA. Silencing the expression of EZH2 led to decreased expression of CCND1 and Ki67 and increased expression of E-cadherin, as determined by western blot analysis. Thus, it was shown that CRAds armed with EZH2 shRNA exhibited significant antitumor effects in human PCa cells. Ad-hTERT-EZH2shRNA may be developed as a treatment for hormone-refractory PCa.

\section{Introduction}

Prostate cancer (PCa) is a type of cancer specifically diagnosed in males, with higher incidence in European and American countries (1). Androgen deprivation therapy (ADT) is the standard of treatment for patients with advanced/metastatic PCa (2). However, patients may inevitably develop castration-resistant prostate cancer (CRPC), which has a poor prognosis (3). Therefore, it is necessary to identify novel molecular mechanisms that regulate cancer progression and develop targeted therapies to improve PCa patient outcomes.

Gene therapy with conditionally replicative adenovirus (CRAds) is an emerging experimental therapeutic strategy for treating refractory cancer resistant to treatment. CRAds appear to be an effective option for targeting androgen-independent prostate cancer. CRAds exert intrinsic anticancer activity through selective replication, resulting in target tumor cell death (4).

Enhancer of zeste homolog 2 (EZH2) is a member of the polycomb group $(\mathrm{PcG})$ protein family, whose main role is to remodel the structure of chromatin. Polycomb repressor complex (PRC) 1 and PRC2 are classified according to their functions. EZH2 is one of the core enzymatic subunits of $\mathrm{PRC} 2$, that methylates histone $\mathrm{H} 3$ at lysine 27 to interfere with the transcription of several genes (5-7). EZH2 is overexpressed in many cancers. It has been reported that multiple gain or loss-of-function EZH2 mutations occur in distinct cancer types (8), including myelodysplastic syndromes (MDS), breast cancer, and PCa. EZH2 is not only a key epigenetic 
inhibitor of histone methylation, but also a gene expression activator through different pathways (9). Many studies have highlighted the association between EZH2 expression and PCa development. Varambally et al (10) demonstrated a positive association between EZH2 protein expression and PCa invasiveness $(7,11)$. Following radical prostatectomy, EZH2 overexpression is associated with both metastasis and higher risk of $\mathrm{PCa}$ recurrence (12). Furthermore, it has been reported that $\mathrm{EZH} 2$ overexpression is a common phenomenon in $\mathrm{PCa}$ that is associated with a poor clinical outcome in $\mathrm{PCa}$ patients $(13,14)$. Therefore, EZH2 was proposed to be an oncogene, and its increased expression may be used as a marker of prostate tumors with aggressive and metastatic potential (8). These studies demonstrate the role of EZH2 in PCa invasiveness, and suggest that EZH2 may be an effective therapeutic target in PCa therapy. Therefore, the elucidating the mechanisms that regulate $\mathrm{EZH} 2$ function may provide therapeutic insights into the treatment of this cancer.

The catalytic component of human telomerase reverse transcriptase (hTERT) is not expressed in the majority of primary somatic human cells, whereas most cancer cells reactivate telomerase by transcriptional upregulation of Htert (15). It has been demonstrated that the hTERT promoter can be used to restrict gene expression of E1-deleted replication defective adenoviral vectors to telomerase-positive cancer cells (16).

In the present study, the generation of a new adenovirus Ad-hTERT-EZH2 small hairpin (sh)RNA was reported, in which EZH2 shRNA expression cassettes containing the hTERT promoter were inserted. It was found that the Ad-hTERT-EZH2shRNA showed excellent antitumor efficacy on $\mathrm{PCa}$ in vitro. The results suggested that Ad-hTERT-EZH2shRNA may be a promising agent for the treatment of PCa.

\section{Materials and methods}

Tissue specimens. CRPC and benign prostate hyperplasia (BPH) specimens were obtained from transurethral resection of the prostate procedures, following the occurrence of lower urinary tract symptoms. Androgen-dependent prostate cancer (ADPC) samples were obtained from radical prostatectomies. No other treatments, including ADT, radiotherapy and chemotherapy, were performed. Clinical pathological data were obtained from the clinical medical college of Yangzhou University from January 1, 2014 to December 31, 2018. In this prospective study, 120 patients aged 62-77 were enrolled, including $10 \mathrm{CRPC}$ cases, $60 \mathrm{ADPC}$ cases and $50 \mathrm{BPH}$ cases. All of the tissue specimens were confirmed by two pathologists. The present study was approved by the Institutional Review Board of Subei People's Hospital of Jiangsu Province and patient consent was obtained prior to tissue collection.

CRAd construction. The hTERT promoter sequence (Table I) was obtained from the relevant reference (17). The conventional cytomegalovirus (CMV) promoter was replaced with a hTERT promoter in the adenovirus plasmid with green fluorescence. Ad-hTERT-EZH2shRNA was constructed by encoding EZH2-shRNA, which had the following sequences: shRNA1, 5'-GCTAGGTTAATTGGGACCAAA-3'; shRNA2, 5'-GGATGGTACTTTCATTGAAGA-3'; negative control,
5'-TTCTCCGAACGTGTCACGT-3' (17). The construction of CRAds was as described previously (18). The other two complexes were also constructed to act as controls (Ad-CMV and Ad-CMV-EZH2-shRNA). All plasmid constructs were confirmed by detecting the DNA sequences (19).

Immunohistochemistry assay. All specimens were fixed in $4 \%$ formaldehyde at room temperature for $6 \mathrm{~h}$ following excision. Paraffin sections were dewaxed by xylene and hydrated in a gradient ethanol series. All immunohistochemical tissue sections were evaluated by two independent pathologists. Immunohistochemistry was performed with a primary antibody against human EZH2 (cat. no. 3147S; 1:200; Cell Signaling Technology, Inc.) at $4^{\circ} \mathrm{C}$ overnight. The secondary antibody (anti mouse IgG; cat. no. 7076P2; 1:2,000; Cell Signaling Technology, Inc.) was applied at $37^{\circ} \mathrm{Cfor} 15 \mathrm{~min}$. For EZH2 expression scoring, the intensity of positive signal was evaluated by eye, as follows: 0 , no staining; 1 , low staining; 2 , medium staining; and 3, strong staining (20). The samples were then categorized into two groups according to the scores assessed: <2, low expression; 2-3, high expression.

Cell culture. Human PCa cell lines (PC3 and DU145) were provided by the Stem Cell Bank, Chinese Academy of Science (Shanghai, China). Cells were cultured at $37^{\circ} \mathrm{C}$ with $5 \% \mathrm{CO}_{2}$ in RPMI-1640 (HyClone Laboratories; GE Healthcare Life Sciences), $10 \%$ fetal bovine serum (FBS; HyClone Laboratories; GE Healthcare Life Sciences), $50 \mathrm{IU} / \mathrm{ml}$ penicillin and $50 \mathrm{mg} / \mathrm{ml}$ streptomycin (Beyotime Institute of Biotechnology).

Transient transfection. Cells were cultured at $37^{\circ} \mathrm{C}$ overnight in 6-well plates for $24 \mathrm{~h}$, until 50-70\% confluence was reached. The frozen virus was thawed in an ice bath, prior to transfection on the 2 nd day. The virus was diluted to the desired density according to the preliminary experiment $\left(1 \times 10^{10} \mathrm{pfu} / \mathrm{ml}\right)$. Cells and virus were mixed and incubated at $37^{\circ} \mathrm{C}$ for $2 \mathrm{~h}$. Next, the medium was replaced with fresh medium and cells were incubated for 24-36 h before infection was observed by fluorescence microscopy. Transfection efficiency was confirmed by western blotting. Cell proliferation and invasion were assayed following transfection.

Western blotting. Western blot analysis was performed as described previously (21). Cells were collected and lysed in radioimmunoprecipitation assay (RIPA) buffer and the protein samples $(50 \mu \mathrm{g})$ were separated by $10 \%$ SDS-PAGE and electrophoretically transferred to a polyvinylidene difluoride membrane. The membrane was incubated with the following primary antibodies at $4^{\circ} \mathrm{C}$ overnight: EZH2 (dilution 1:1,000; cat. no. 3147S; Cell Signaling Technology, Inc.), E-cadherin (dilution 1:1,000; cat. no. 4A2C7; Thermo Fisher Scientific, Inc.), Cyclin D1 (CCND1; dilution 1:1,000; cat. no. A0310; Shenzhen Yinji Technology, Co., Ltd.) and proliferation marker protein Ki-67 (dilution 1:1,000; cat. no. 14-5698-82; Cell Signaling Technology, Inc.), followed by secondary antibody (dilution 1:2,000; cat. no. 7076P2; Cell Signaling Technology, Inc.) incubation at room temperature for $2 \mathrm{~h}$. Finally, signal detection was performed by chemiluminescence using an ECL kit (Thermo Fisher Scientific, Inc.). GAPDH (dilution 1:10,000; Santa Cruz Biotechnology, Inc.) was used as a 
Table I. Sequences of hTERT promoter.

\begin{tabular}{ll}
\hline Name & \multicolumn{1}{c}{ Sequences } \\
\hline h-TERT & GGCCCCTCCCTCGGGTTACCCCACAGCCTAGGCCGATTCGACCTCTCTCCGCTGGGGCCCTCGCTGG \\
& CGTCCCTGCACCCTGGGAGCGCGAGCGGCGCGCGGGCGGGGAAGCGCGGCCCAGACCCCCGGGTC \\
& CGCCCGGAGCAGCTGCGCTGTCGGGGCCAGGCCGGGCTCCCAGTGGATTCGCGGGCACAGACGCCC \\
& AGGACCGCGCTCCCCACGTGGCGGAGGGACTGGGGACCCGGGCACCCGTCCTGCCCCTTCACCTTC \\
& CAGCTCCGCCTCCTCCGCGCGGACCCCGCCCCGTCCCGACCCCTCCCGGGTCCCCGGCCCAGCCCC \\
& CTCCGGGCCCTCCCAGCCCCTCCCCTTCCTTTCCGCGGCCCCGCCCTCTCCTCGCGGCGCGAGTTTC \\
& AGGCAGCGCTGCGTCCTGCTGCGCACGTGGGAAGCCCTGGCCCCGGCCACCCCCGCG
\end{tabular}

hTERT, human telomerase reverse transcriptase.

protein loading control. Signals were visualized by using a Molecular Image ChemiDocÔ XRS+ system with Image Lab software version 5.2.1 (Bio-Rad Laboratories, Inc.).

Reverse transcription-quantitative polymerase chain reaction $(R T-q P C R)$. RT-qPCR assays were used to detect EZH2 transcript expression. According to the manufacturer's instructions, total RNA was extracted from cell samples using TRIzol reagent (Thermo Fisher Scientific, Inc.), and $1 \mu \mathrm{g}$ total RNA was reverse transcribed into cDNA using SuperScript II reverse transcriptase (cat. no. 18090050; Thermo Fisher Scientific, Inc.). EZH2 and internal control GAPDH were then amplified by qPCR using the following primers: EZH2 forward, 5'-CCA AGAGAGCCATCCAGACT-3' and reverse, 5'-CGATGCCGA CATACTTCAGG-3'; GAPDH forward, 5'-GCATCAAGG GAGACACCA-3' and reverse, 5'-TGACCTAACTAAAGC ACCAGA-3' (22). qPCR was conducted by using the Step One PlusÔ Real-Time PCR system (Thermo Fisher Scientific, Inc., Waltham, MA, USA). All samples were amplified in triplicate. The PCR thermocycling conditions were as follows: $95^{\circ} \mathrm{C}$ for $30 \mathrm{sec}$, then 40 cycles of $10 \mathrm{sec}$ at $95^{\circ} \mathrm{C}, 60^{\circ} \mathrm{C}$ for $20 \mathrm{sec}$ and $70^{\circ} \mathrm{C}$ for $10 \mathrm{sec}$. The $2^{-\Delta \Delta \mathrm{Cq}}$ method was used to calculate relative gene expression (23).

Transwell Matrigel invasion assay. Cell invasion ability was detected in 24-well plates. Matrigel (BD Biosciences, Franklin Lakes, NJ, USA) was diluted to 8-fold with serum-free medium, which was then injected into the upper chambers with polycarbonate membranes $(8 \mu \mathrm{m}$ pore size) the night before the experiment. Next, transfected PC3 and DU145 cells were diluted to $5 \times 10^{4} / \mathrm{ml}$ and added into the upper chamber with serum-free medium $(200 \mu 1)$, and the lower chambers were filled with $200 \mu 1$ complete medium (RPMI-1640 with $10 \% \mathrm{FBS})$. Cells were cultured for $12 \mathrm{~h}$ at $37^{\circ} \mathrm{C}$ at an atmosphere of $5 \% \mathrm{CO}_{2}$. Non-invading cells were removed from the top of the filter and invaded cells were fixed with $100 \%$ methanol for $30 \mathrm{~min}$ at room temperature. Crystal violet $(0.1 \%)$ was used to stain the cells at room temperature for $10 \mathrm{~min}$ and the invaded cell number was counted in 10 random fields of view using an inverted light microscope.

Cell Counting Kit (CKK)-8 assay. Cell growth was analyzed by using a WST-8 Cell Counting Kit-8 (Dojindo Molecular Technologies, Inc., Shanghai, China). Cells $\left(2.5 \times 10^{3}\right)$ suspended in RPMI-1640 medium containing $10 \%$ FBS were seeded in 96-well plates and incubated for 24, 48 and $72 \mathrm{~h}$, respectively. CCK-8 solution $(10 \mu \mathrm{l})$ was added to each well for $30 \mathrm{~min}$ at $37^{\circ} \mathrm{C}$. Absorbance was subsequently measured at $450 \mathrm{~nm}$ with a microplate reader.

Statistical analysis. Statistical analyses were performed with SPSS version 25.0 (IBM Corp., Armonk, NY, USA). Values are presented as the mean \pm standard deviation from at least three independent experiments. Differences were assessed by Student's t-test or one-way analysis of variance followed by Tukey's post hoc test. Recurrence survival was analyzed using the Kaplan-Meier method. $\mathrm{P}<0.05$ was considered to indicate a statistically significant difference.

\section{Results}

Clinical relevance of EZH2 expression in human PCa specimens. First, the expression of EZH2 in BPH, ADPC and CRPC patients was examined by immunohistochemistry. Compared with the BPH specimens, the expression of EZH2 was markedly upregulated in ADPC and CRPC specimens (Fig. 1A). The expression of EZH2 was associated with the category of specimens $(\mathrm{P}<0.01$; Fig. 1B). After 18-30 months on ADT, large amounts of patients of ADPC progress to CRPC, particularly in patients whose EZH2 intensity scores were equal to $3(\mathrm{P}<0.05$; Fig. 1C). The time of progression-free survival in ADPC patients with EZH2 scores $<2$ was significantly increased compared to patients with EZH2 scores $\geq 2$ ( $\mathrm{P}<0.05$; Fig. 1D).

Successful construction of conditionally replicative adenovirus. Following CRAd construction, shRNA and hTERT promoter expression was identified in the monoclonal colonies by sequencing (Fig. 2A-C). For the hTERT promoter, the arrow symbol indicated the reverse complementary sequence from the beginning to the end. The correct clone was the successfully constructed target gene of adenovirus vector. CCK-8 assays were used to detect the transfection efficiency of the two shRNAs in the PC3 cell line. shRNA2 exhibited better inhibition of PC3 proliferation (Fig. 2D). As EZH2 and its protein are overexpressed in human lung adenocarcinoma A549 cells (24), adenovirus was transfected into these cells, which were then observed under a microscope $24 \mathrm{~h}, 48 \mathrm{~h}$ and $72 \mathrm{~h}$ after transfection. The fluorescence indicated that the 
A

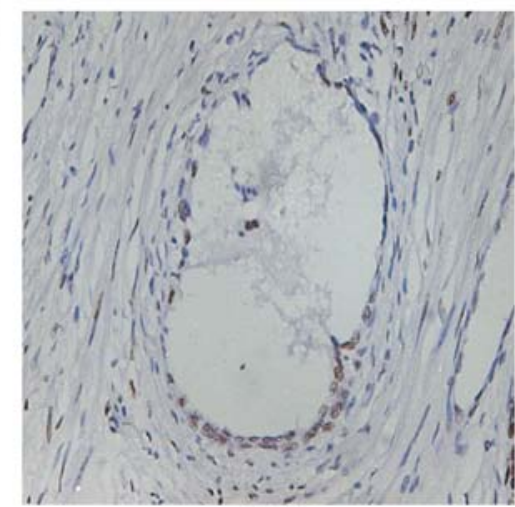

$\mathrm{BPH}$

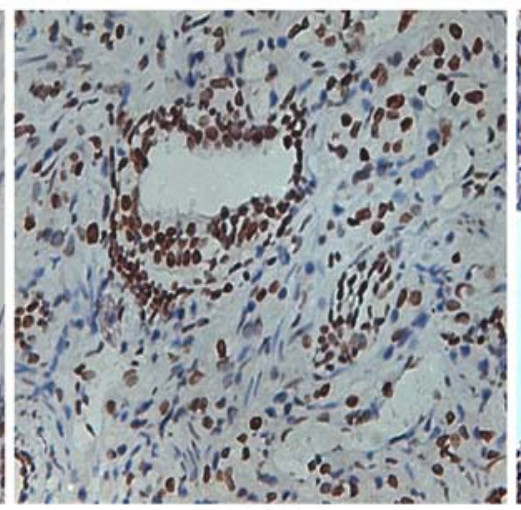

ADPC

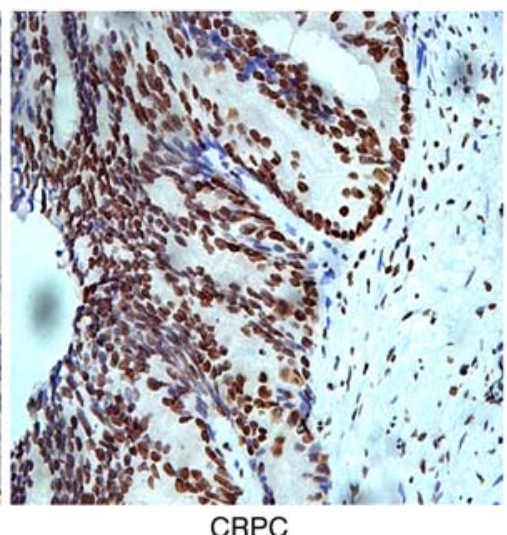

CRPC
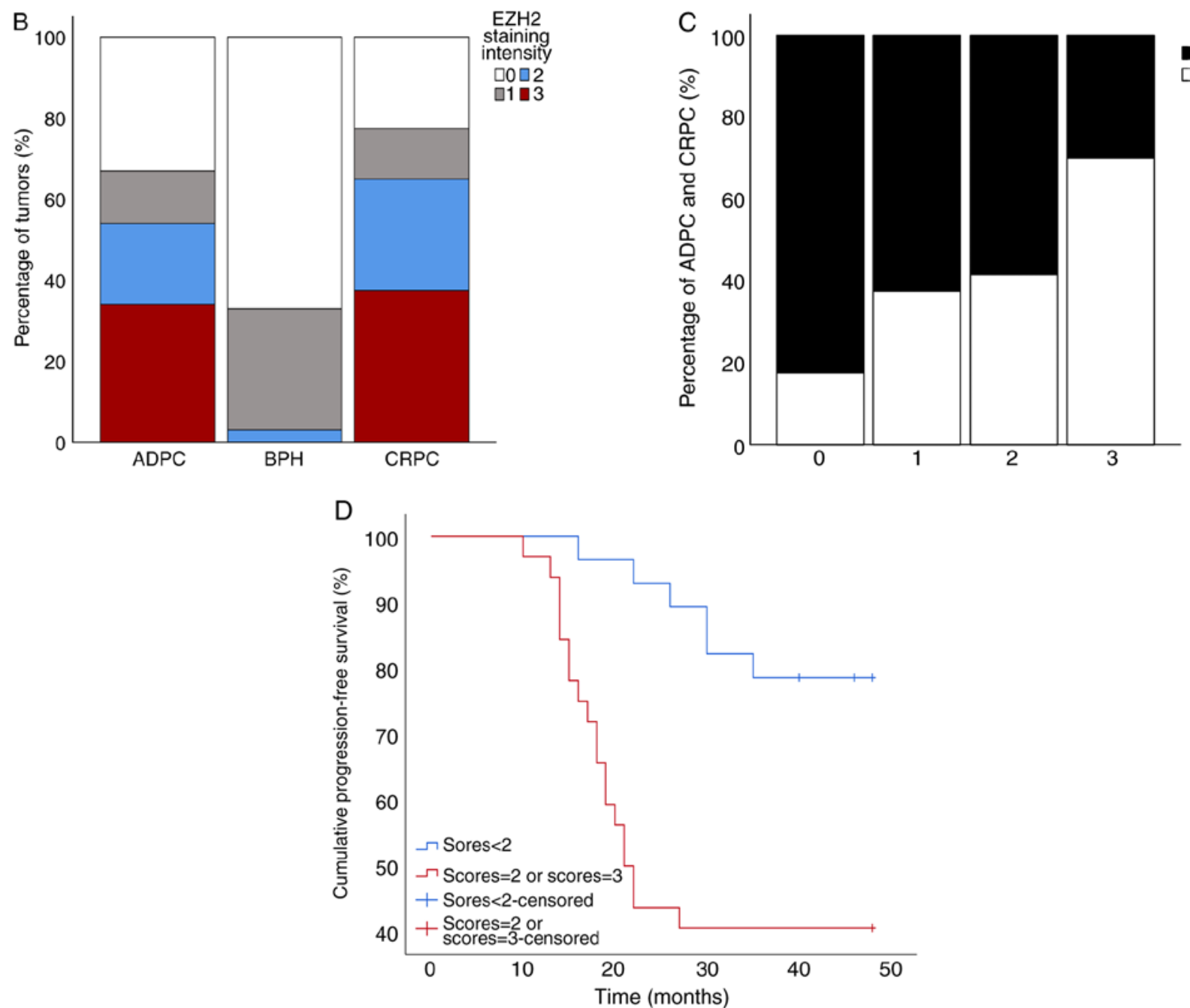

Figure 1. EZH2 expression in BPH, ADPC and CRPC specimens. (A) Immunohistochemical analysis of EZH2 in BPH, ADPC and CRPC specimens (original magnification, $\mathrm{x} 400$ ). (B) Scoring of the staining intensities indicated that EZH2 protein expression was significantly increased in ADPC and CRPC specimens, compared with the BPH tissues. (C) After 18-30 months of ADT, the majority of patients progressed from CRPC to ADPC. (D) Kaplan-Meier analyses of cumulative progression-free survival compared with the scores of the staining intensities $(\mathrm{P}<0.05)$. EZH2, enhancer of zeste homolog 2 ; BPH, benign prostate hyperplasia; ADPC, androgen-dependent prostate cancer; CRPC, castration-resistant prostate cancer; ADT, androgen deprivation therapy.

transfected cells increased gradually with the extension of time which indirectly represented that adenovirus vector was successfully constructed (Fig. 2E).

EZH2 expression is reduced by Ad-hTERT-EZH2shRNA. PCa cells (PC3 and DU145) were transfected with three types of adenoviruses, respectively. After $72 \mathrm{~h}$ of transfection, the expression of EZH2 was detected by western blotting (Fig. 3A). The results suggested that Ad-hTERT-EZH2shRNA (Test group) transfection most significantly inhibited the expression of EZH2 gene in PCa cells, followed by Ad-CMV-EZH2shRNA (CMV-shRNA). RT-qPCR also showed that Ad-hTERT-EZH2shRNA significantly reduced the gene expression of EZH2 (Fig. 3B). 


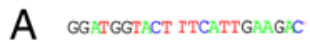

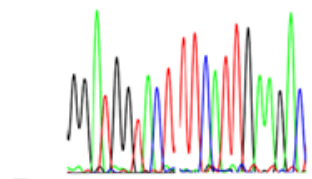

C

GCTAGTIAMTGGG:CCNAP
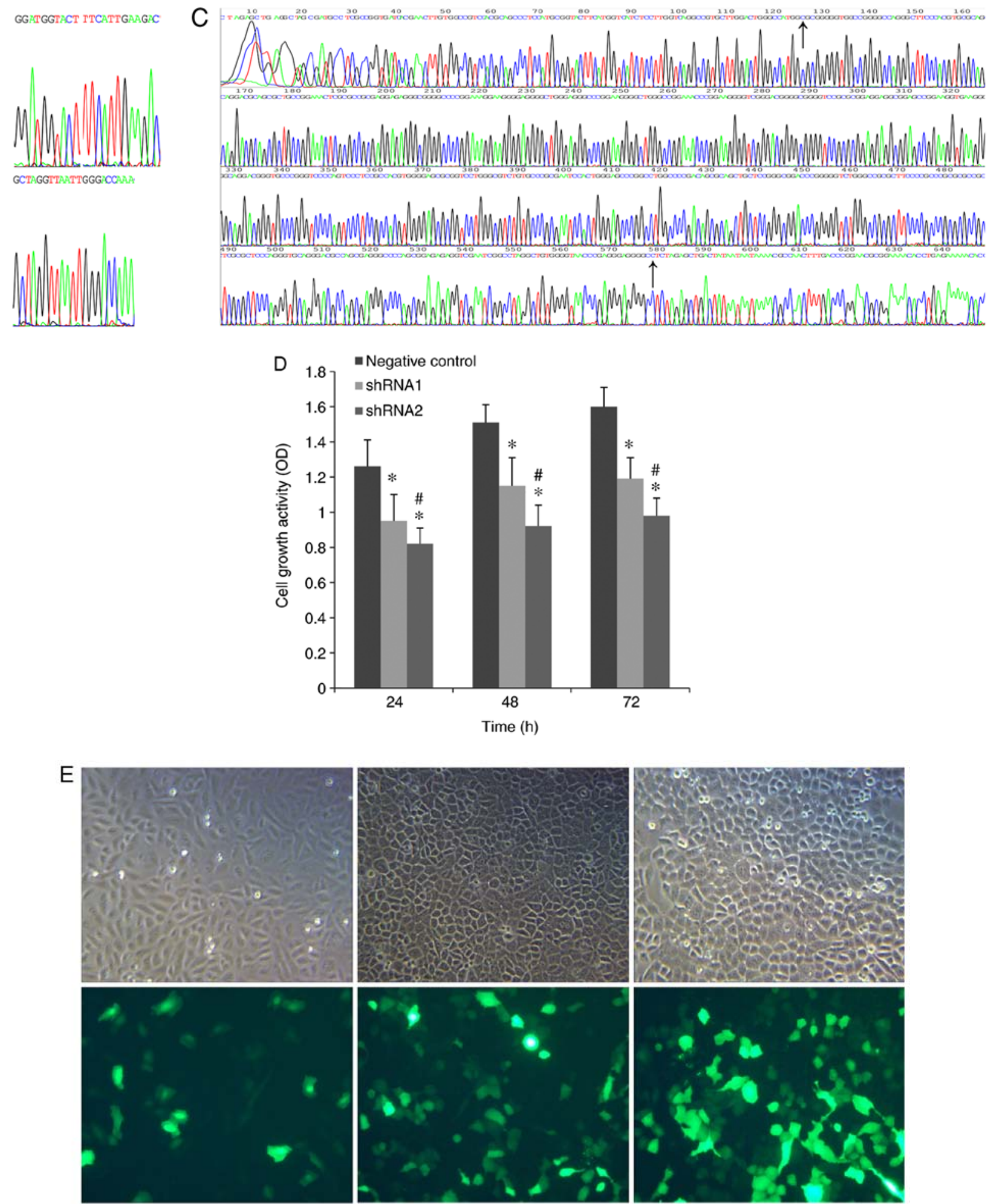

$24 \mathrm{~h}$

$48 \mathrm{~h}$

$72 \mathrm{~h}$

Figure 2. Successful construction of the CRAds vectors. (A and B) Cultured monoclonal colonies were sent to the sequencing company for EZH2-shRNA sequence identification. (C) The sequence identification of hTERT (reverse complementary sequencing). (D) Cell proliferation was measured at three time points with Cell Counting Kit-8 assays in the control, shRNA1 and shRNA2 transfected PC 3 cells $\left(24,48\right.$ and 72 h). ${ }^{*} \mathrm{P}<0.05$ vs. control; ${ }^{~} \mathrm{P}<0.05$ vs. shRNA1. (E) A549 cells transfected with CRAds were observed under a fluorescence microscope after 24,48 and $72 \mathrm{~h}$ (original magnification, x100). CRAds, conditionally replicative adenovirus; EZH2, enhancer of zeste homolog 2; shRNA, small hairpin RNA; hTERT, human telomerase reverse transcriptase.

Cell invasion assay. To examine the invasion ability of the PCa cells, Transwell assays were performed following cell transfection and compared with the negative control group. Ad-hTERT-EZH2shRNA transfected cells ex hibited a decrease in cell invasion after culture for $48 \mathrm{~h}$, respectively (Fig. 4).
Cell proliferation assay. To examine the tumor-suppressive function of EZH2, the effect of EZH2 knockdown on cell proliferation was measured using CCK-8 assays. Following transfection, shRNA was stably expressed in PCa cell lines, compared with the negative control. Transfection with 
A
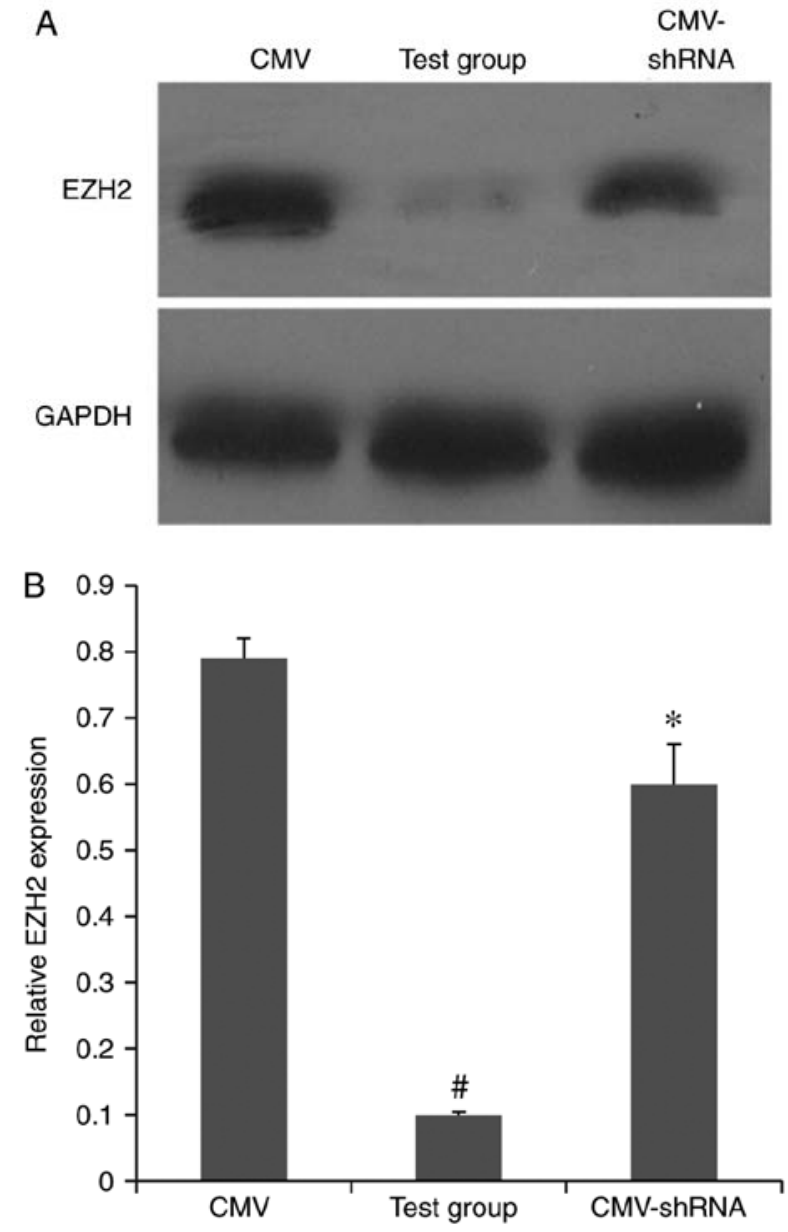

Figure 3. EZH2 expression is reduced by Ad-hTERT-EZH2shRNA. (A) Western blot analysis of EZH2 expression in cells transfected with three types of CRAds. (B) Reverse transcription-quantitative polymerase chain reaction analysis of EZH2 expression in the three experimental groups. ${ }^{*} \mathrm{P}<0.05$ vs. CMV group; ${ }^{*} \mathrm{P}<0.05$ vs. $\mathrm{CMV}$-shRNA group. $\mathrm{CMV}$, cytomegalovirus; EZH2, enhancer of zeste homolog 2; shRNA, small hairpin RNA hTERT, human telomerase reverse transcriptase.

EZH2-shRNA resulted in a significant decrease of cell growth in the $\mathrm{PCa}$ cell lines $(\mathrm{P}<0.05$; Fig. 5A and $\mathrm{B})$.

EZH2 knockdown upregulates E-cadherin and represses Ki67 and CCND1 expression in PCa cell lines. In order to explore the mechanism of EZH2 in $\mathrm{PCa}$, the relationship between EZH2, E-cadherin, Ki67 and CCND1 was examined. The expression of EZH2, E-cadherin, Ki67 and CCND1 was measured by western blotting following transfection with Ad-hTERT-EZH2shRNA. Untreated cells were used as a negative control. The expression level of EZH2, Ki67 and CCND1 was decreased by shRNA, whereas E-cadherin expression was repressed (Fig. 6A and B).

\section{Discussion}

Despite advances in technology that have allowed earlier diagnoses, PCa morbidity remains high, particularly in CRPC cases. CRPC occurs after 18 to 30 months of ADT (3). Limited therapeutic options are available once androgen resistance develops. Therefore, increasing amount of research has focused on gene therapy for patients with CRPC (25).
Oncolytic viral therapy is a novel method of tumor treatment, where tumor cells are selectively infected with viruses, resulting in tumor cell death and stimulation of a specific anti-tumor immune response (26-28). Oncolytic viruses are modified with attenuated or genetically engineered viruses, which are highly specific to tumor cells, meaning they have limited effects on normal tissues (29). The main side effects are influenza-like symptoms (<grade 3). In nearly 100 clinical studies of oncolytic viruses, no serious side effects caused by viruses have been found. No dose limiting toxicity was observed, and the safety was reliable (30).

Due to their stability and well-established methodology, traditional transgenic and gene targeting technologies currently remain the main strategy used for model construction, rather than the CRISPR/Cas system (31). CRAds selectively replicate and spread within tumor cells, meaning this tool not only has a strong tumor scavenging ability, but can also be used in reduced doses. This consequently reduces any toxic liver effects of the liver, and thus CRAds have gradually become a widespread concern in gene therapy (31).

EZH2 serves a pivotal role in regulating chromosomal structure (32). High EZH2 expression has been detected in a variety of tumors and is associated with the degree of tumor malignancy, invasion and metastasis $(33,34)$. Varambally (10) et al showed that EZH2 siRNA transfection markedly inhibited benign prostate cell growth. A previous study also reported that EZH2 may be a potential target in the treatment of PCa (8). In the present study, it was demonstrated that EZH2 staining was localized in the nucleus of tumor cells, and EZH2 was overexpressed in PCa tissues compared to BPH. EZH2 shRNA transfection reduced the RNA and protein expression of EZH2, as determined by RT-qPCR and western blot analysis. In addition, EZH2 gene expression is low in early stage PCa, but significantly increased in CRPC, compared with $\operatorname{ADPC}(35,36)$. EZH2 knockdown inhibits cancer cell growth and metastasis $(37,38)$. Studies have confirmed that EZH2 is positively correlated with tumor angiogenesis, as EZH2 is involved in the formation of VEGF $(39,40)$. Researchers also found that in ADPC, EZH2 binds to androgen receptors as an androgen receptor transcription factor, in place of androgen, thereby contributing to the progression of PCa (9). In the present study, the effects of EXH2 on PCa cell growth and invasiveness was demonstrated in two CRPC cell lines. This indicated that EZH2 shRNA had potential anti-CRPC tumor activity.

Studies have shown that the aberrant expression of EZH2 in $\mathrm{PCa}$ at an early stage may promote the progression of PCa into a more aggressive form (36). In the present study, decreased EZH2 expression reduced $\mathrm{PCa}$ cell proliferation and invasion. Taken together, these findings indicate that EZH2 is involved in the proliferation, invasion, progression, metastasis of $\mathrm{PCa}$ cells $(41,42)$.

Telomerase is positively expressed in $>90 \%$ of human tumor cells but absent in almost all of the normal cells (43). hTERT is a determinant factor in telomerase activity among human telomerase components. hTERT is highly expressed in $~ 90 \%$ of malignant tumors, but inactive in normal cells $(44,45)$. Research has shown that hTERT activity levels 
A
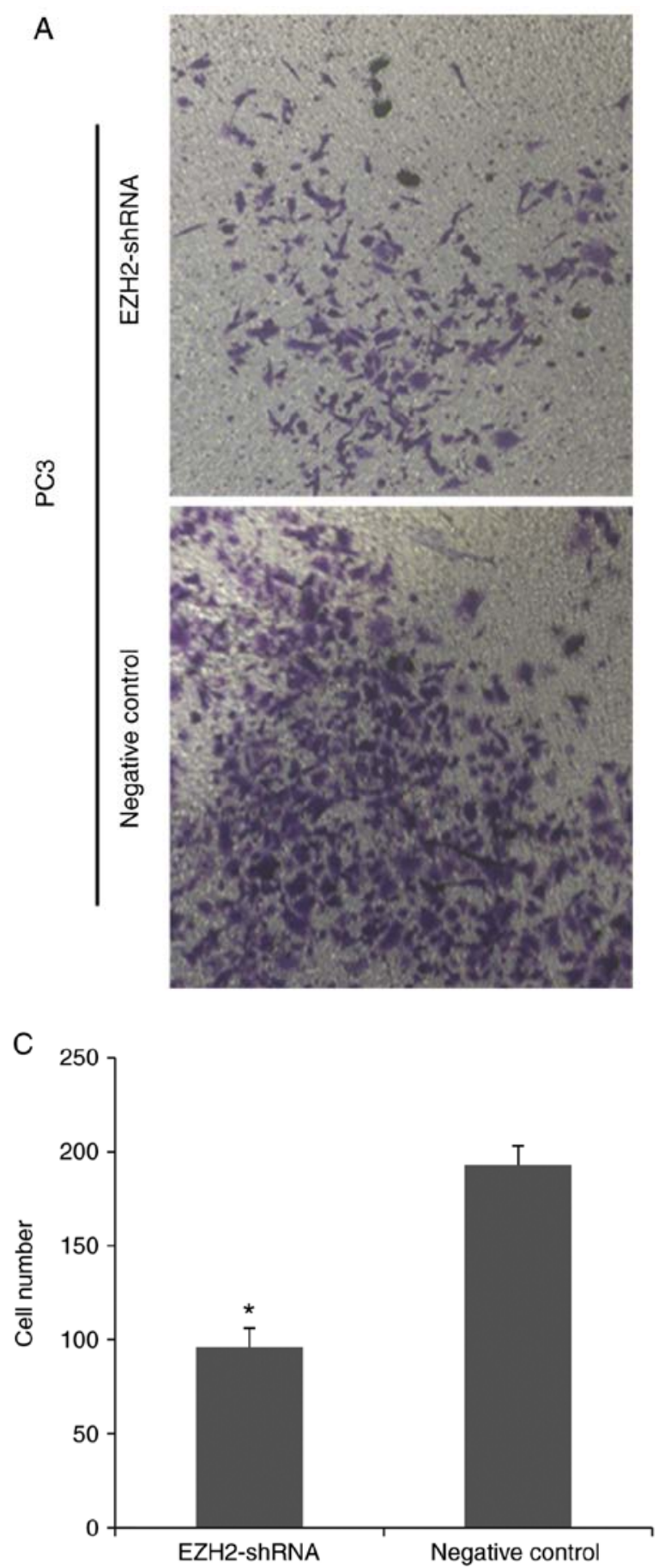

B
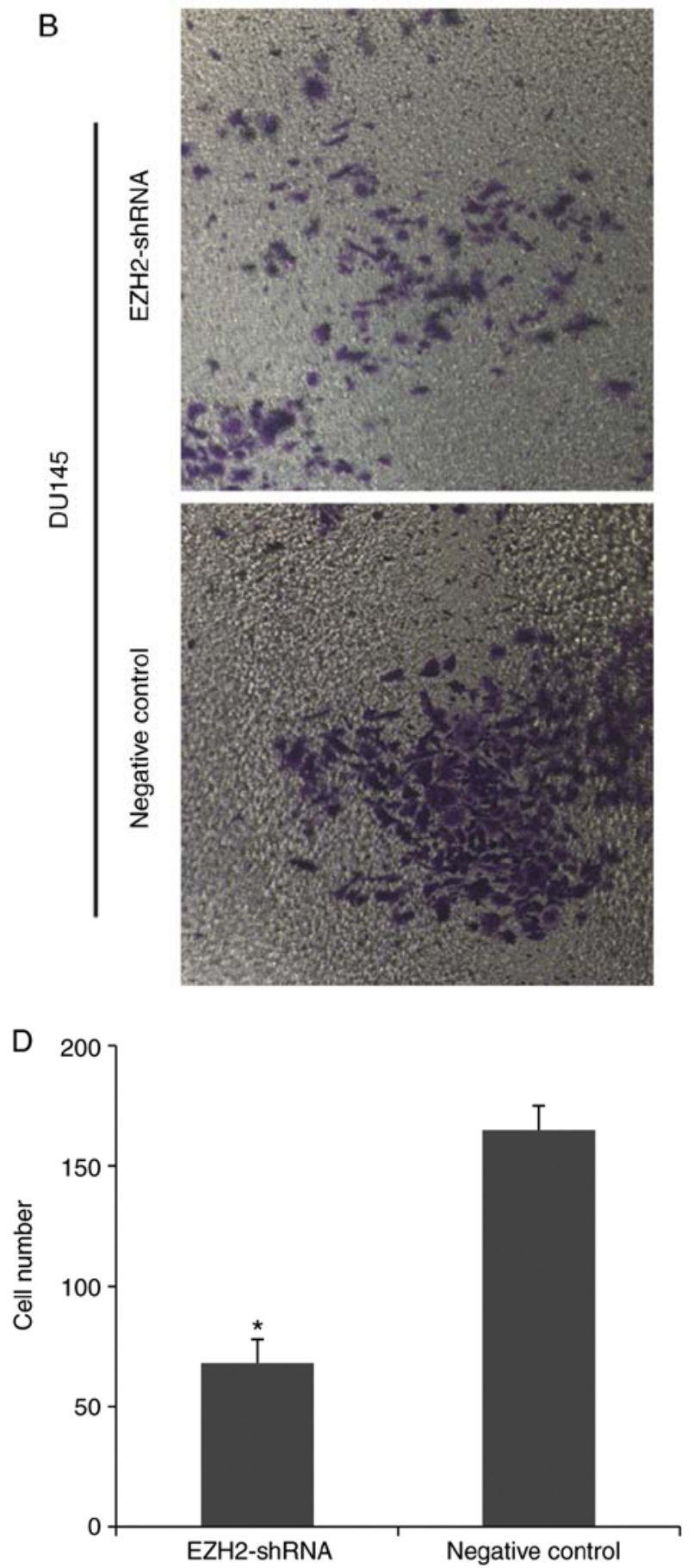

Figure 4. EZH2 promotes the invasion of prostate cancer cells. (A and B) Transwell assay analysis the invasion capacity of (A) PC3 and (B) DU145 cells (original magnification, $\mathrm{x} 100$ ). (C) PC3 and (D) DU145 cell number across the chamber was determined after culturing for $12 \mathrm{~h}$. * $\mathrm{P}<0.05$ vs. negative control. EZH2, enhancer of zeste homolog 2 .

exhibit a prevalence range of $63-94 \%$ for PCa (46). Vectors carrying the hTERT promoter have a targeted effect on malignant tumors $(47,48)$. Based on the information above, hTERT was selected as a core promoter in the present study, in order to drive a specific target sequence in CRAds so that it could specifically be expressed in PCa tissues, but not in normal tissues. Western blotting showed that the experimental group (Ad-hTERT-EZH2shRNA) had obvious advantages in inhibiting the expression of EZH2 compared to the other two controls, and that the hTERT promoter was more effective in delivering the EZH2-shRNA.
Patients with the same PCa stage or grade often exhibit different clinical features, making it difficult to accurately predict prognosis with clinical stage and pathology data alone. In the present study, the expression of Ki67, E-cadherin and CCND1 may have also been associated with both PCa development and recurrence $(5,49,7)$. E-Cadherin is a classical member of the cadherin superfamily. Loss of function is thought to contribute to progression in cancer by increasing proliferation, invasion and/or metastasis $(50,51)$. Antigen $\mathrm{Ki67}$ is a nuclear protein that is associated with and may be necessary for cellular proliferation. Furthermore, it is 

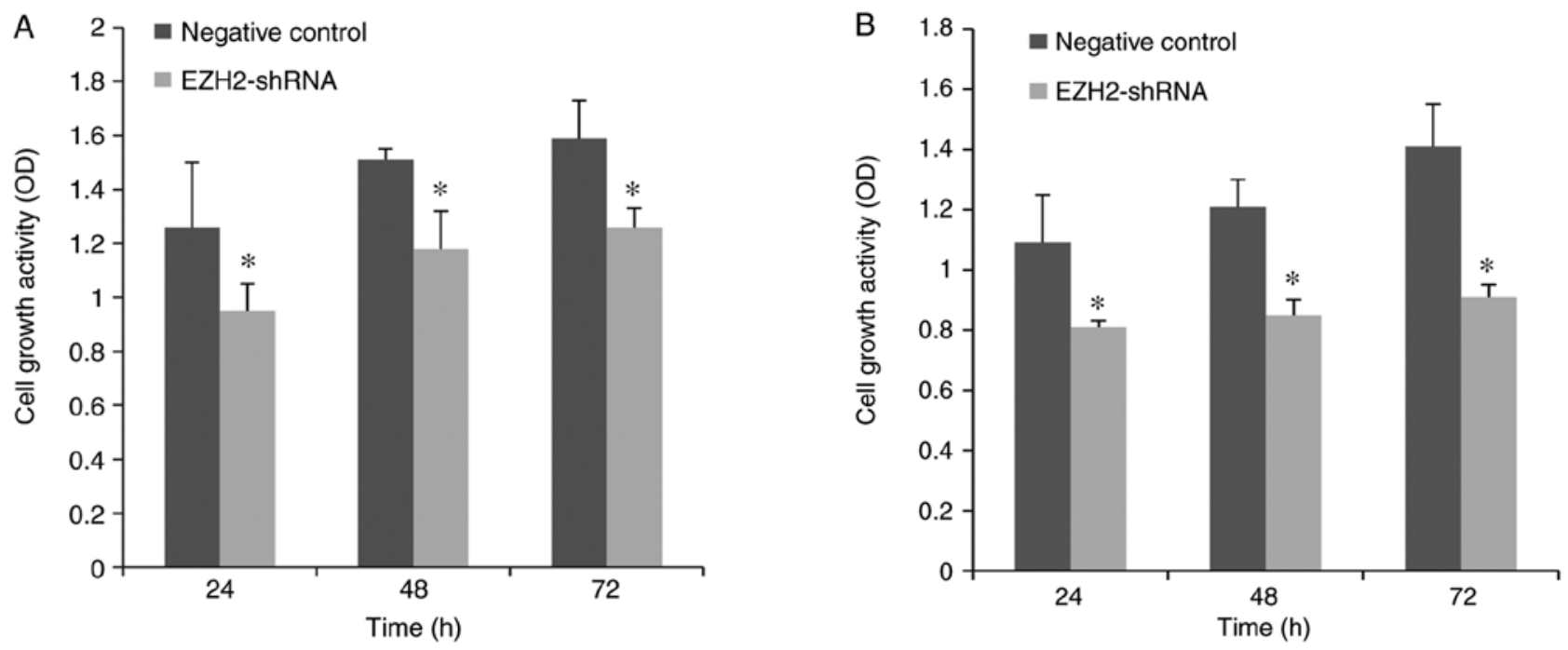

Figure 5. EZH2-shRNA inhibits the proliferation of PC 3 and DU145 cells. Cell proliferation was measured at 24,48 and $72 \mathrm{~h}$ with Cell Counting Kit- 8 assays in the control and EZH2-shRNA transfected (A) PC3 and (B) DU145 cells. " P<0.05 vs. negative control. EZH2, enhancer of zeste homolog 2; shRNA, small hairpin RNA.

A
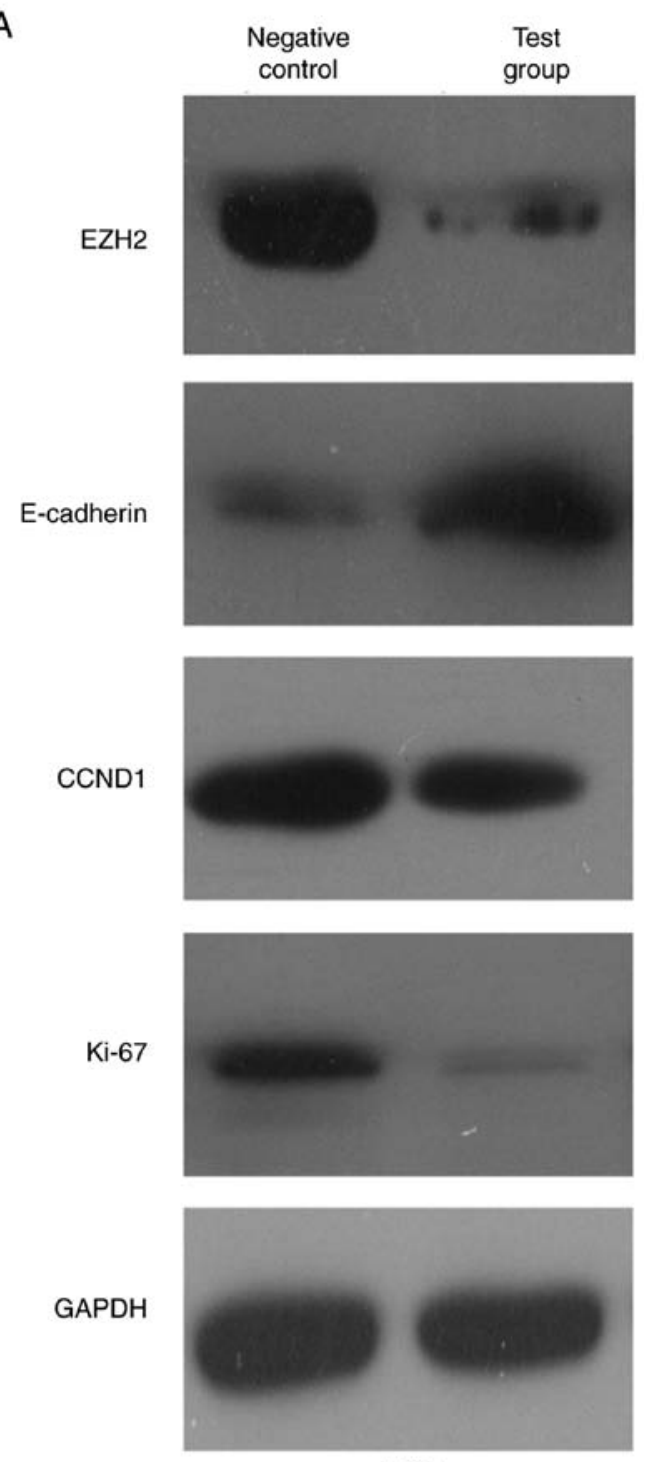

PC3
B
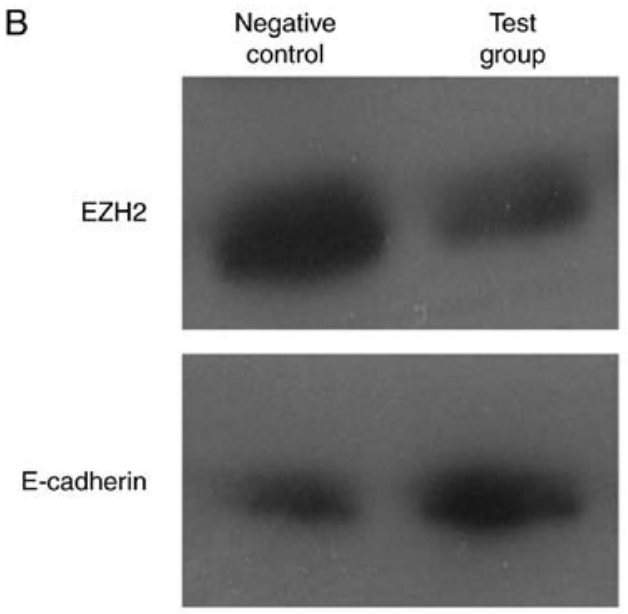

CCND1

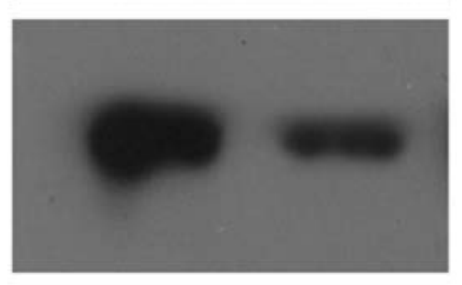

$\mathrm{Ki}-67$

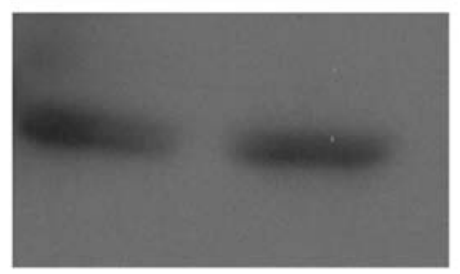

GAPDH

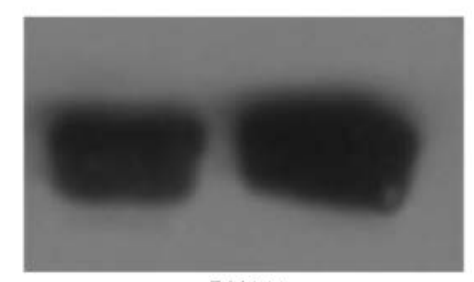

DU145

Figure 6. EZH2 knockdown upregulates E-cadherin and represses Ki67 and CCND1 expression in prostate cancer cell lines. (A and B) EZH2, E-cadherin, CCND1 and Ki67 expression was detected by western blotting in (A) PC3 and (B) DU145 cells. EZH2, enhancer of zeste homolog 2; CCND1, cyclin D1; $\mathrm{Ki} 67$, proliferation marker protein Ki67. 
associated with ribosomal RNA transcription (52). Ki67 is an excellent marker to determine the growth fraction of a given cell population. The fraction of Ki67-positive tumor cells is often correlated with the clinical outcome in cancer. Finally, the main function of CCND1 is to promote cell proliferation. A previous study has demonstrated that CCND1 significantly decreased in EZH2-deficient cells (53). In the present study, the results indicated that silencing EZH2 led to decreased expression of CCND1 and Ki67, and increased the expression of E-cadherin. When combined with other clinicopathological information, such as pathological grade, CCND1, Ki67 and E-cadherin could be used as prognostic biomarkers. However, the signaling pathways between EZH2, CCND1, Ki67 and E-cadherin remain unclear. The underlying mechanism should be investigated in our future studies.

The current study provided evidence that EZH2 was correlated with CRPC cell invasion and proliferation, but there are several limitations. First, the number of CRPC specimens obtained was small, and more samples should be used in further studies. Second, an animal model should be established in the future to better demonstrate the efficacy of the Ad-hTERT-EZH2-shRNA. In addition, gene over-expression experiments was absent in the present study, as a large number of studies have proved that the EZH2 gene is abundantly expressed in cancer cells $(8,13)$.

Collectively, the present study showed that CRAds armed with EZH2 shRNA exhibited significant antitumor effects in human PCa cells. EZH2 knockdown suppressed PCa cell proliferation and invasion. Additionally, silencing EZH2 led to the decreased expression of CCND1 and Ki67, and increased E-cadherin expression. To the best of our knowledge, the present study revealed a novel mechanism by which CRAds with EZH2 shRNA inhibited PCa cell growth, and highlighted the potential clinical importance of CRAds in CRPC therapies.

\section{Acknowledgements}

The authors thank the laboratory staff at the Clinical Medical College of Yangzhou University (Subei People's Hospital of Jiangsu Province) for technical support.

\section{Funding}

The present study was supported by a grant from the National Natural Science Foundation of China (grant no. 81402101).

\section{Availability of data and materials}

All data generated or analyzed during this study are included in this published article.

\section{Authors' contributions}

XG, YXW and GCZ designed the study. SGX and JJY wrote the manuscript, collected clinical information and performed statistical analyses; QS, QN, ZG and BYG assisted with immunohistochemical analysis, cell culture, PCR, western blotting and in vitro experiments. All authors read and approved the manuscript and agree to be accountable for all aspects of the research in ensuring that the accuracy or integrity of any part of the work are appropriately investigated and resolved.

\section{Ethics approval and consent to participate}

The present study was approved by institutional Review Board of Clinical Medical College of Yangzhou University (Subei People's Hospital of Jiangsu Province) and patient consent was obtained prior to tissue collection.

\section{Patient consent for publication}

Not applicable.

\section{Competing interests}

The authors declare that they have no competing interests.

\section{References}

1. Siegel RL, Miller KD and Jemal A: Cancer statistics, 2018. CA Cancer J Clin 68: 7-30, 2018.

2. Klil-Drori AJ, Tascilar K, Yin H, Aprikian A, Bitton A and Azoulay L: Androgen deprivation therapy and the incidence of inflammatory bowel disease in patients with prostate cancer. Am J Epidemiol 184: 15-22, 2016

3. Massard C and Fizazi K: Targeting continued androgen receptor signaling in prostate cancer. Clin Cancer Res 17: 3876-3883, 2011.

4. Irving J, Wang Z, Powell S, O'Sullivan C, Mok M, Murphy B, Cardoza L, Lebkowski JS and Majumdar AS: Conditionally replicative adenovirus driven by the human telomerase promoter provides broad-spectrum antitumor activity without liver toxicity. Cancer Gene Ther 11: 174-185, 2004.

5. Cao Q, Yu J, Dhanasekaran SM, Kim JH, Mani RS, Tomlins SA, Mehra R, Laxman B, Cao X, Yu J, et al: Repression of E-cadherin by the polycomb group protein EZH2 in cancer. Oncogene 27: 7274-7284, 2008.

6. Liu L, Xu Z, Zhong L, Wang H, Jiang S, Long Q, Xu J and Guo J: Enhancer of zeste homolog $2(\mathrm{EZH} 2)$ promotes tumour cell migration and invasion via epigenetic repression of E-cadherin in renal cell carcinoma. BJU Int 117: 351-362, 2016.

7. Lobo J, Rodrigues Â, Antunes L, Graça I, Ramalho-Carvalho J, Vieira FQ, Martins AT, Oliveira J, Jerónimo C and Henrique R: High immunoexpression of Ki67, EZH2, and SMYD3 in diagnostic prostate biopsies independently predicts outcome in patients with prostate cancer. Urol Oncol 36: 161.e167-161.e117, 2018.

8. Kim KH and Roberts CW: Targeting EZH2 in cancer. Nat Med 22: 128-134, 2016.

9. Xu K, Wu ZJ, Groner AC, He HH, Cai C, Lis RT, Wu X, Stack EC, Loda M, Liu T, et al: EZH2 oncogenic activity in castrationresistant prostate cancer cells is Polycomb-independent. Science 338: 1465-1469, 2012.

10. Varambally S, Dhanasekaran SM, Zhou M, Barrette TR, Kumar-Sinha C, Sanda MG, Ghosh D, Pienta KJ, Sewalt RG, Otte AP, et al: The polycomb group protein EZH2 is involved in progression of prostate cancer. Nature 419: 624-629, 2002.

11. Xiang S, Zou P, Tang Q, Zheng F, Wu J, Chen Z and Hann SS: HOTAIR-mediated reciprocal regulation of EZH2 and DNMT1 contribute to polyphyllin I-inhibited growth of castrationresistant prostate cancer cells in vitro and in vivo. Biochim Biophys Acta Gen Subj 1862: 589-599, 2018.

12. Laitinen S, Martikainen PM, Tolonen T, Isola J, Tammela TL and Visakorpi T: EZH2, Ki-67 and MCM7 are prognostic markers in prostatectomy treated patients. Int J Cancer 122: 595-602, 2008.

13. Gu X, Gao XS, Bai Y, et al: EZH2 overexpression as a biomarker of poor prognosis in prostate cancer. 2016.

14. Cao P, Deng Z, Wan M, Huang W, Cramer SD, Xu J, Lei M and Sui G: MicroRNA-101 negatively regulates Ezh2 and its expression is modulated by androgen receptor and HIF-1alpha/HIF-1beta. Mol Cancer 9: 108, 2010. 
15. Wirth T, Zender L, Schulte B, Mundt B, Plentz R, Rudolph KL, Manns M, Kubicka S and Kühnel F: A Telomerase-dependent conditionally replicating adenovirus for selective treatment of cancer. Cancer Res 63: 3181-3188 2003.

16. Kirch HC, Ruschen S, Brockmann D, Esche H, Horikawa I, Barrett JC, Opalka B and Hengge UR: Tumor-specific activation of hTERT-derived promoters by tumor suppressive E1A-mutants involves recruitment of $\mathrm{p} 300 / \mathrm{CBP} / \mathrm{HAT}$ and suppression of HDAC-1 and defines a combined tumor targeting and suppression system. Oncogene 21: 7991-8000, 2002.

17. Liu L, Liu Y, Zhang T, Wu H, Lin M, Wang C, Zhan Y, Zhou Q, Qiao B, Sun X, et al: Synthetic Bax-Anti Bcl2 combination module actuated by super artificial hTERT promoter selectively inhibits malignant phenotypes of bladder cancer. J Exp Clinl Cancer Res 35: 3, 2016.

18. Cebrià F, Kobayashi C, Umesono Y, Nakazawa M, Mineta K, Ikeo K, Gojobori T, Itoh M, Taira M, Sánchez Alvarado A and Agata K: FGFR-related gene nou-darake restricts brain tissues to the head region of planarians. Nature 419: 620-624, 2002.

19. Sanger F, Nicklen S and Coulson AR: DNA sequencing with chain-terminating inhibitors. Proc Natl Acad Sci USA 74: 5463-5467, 1977.

20. Ruan J, Wei B, Xu Z, Yang S, Zhou Y, Yu M, Liang J, Jin K, Huang X, Lu P and Cheng H: Predictive value of Sox 2 expression in transurethral resection specimens in patients with $\mathrm{T} 1$ bladder cancer. Med Oncol 30: 445, 2013.

21. Yu J, Lu Y, Cui D, Li E, Zhu Y, Zhao Y, Zhao F and Xia S: miR-200b suppresses cell proliferation, migration and enhances chemosensitivity in prostate cancer by regulating Bmi-1. Oncol Rep 31: 910-918, 2014.

22. Li K, Liu C, Zhou B, Bi L, Huang H, Lin T and Xu K: Role of $\mathrm{EZH} 2$ in the growth of prostate cancer stem cells isolated from LNCaP cells. Int J Mol Sci 14: 11981-11993, 2013.

23. Livak KJ and Schmittgen TD: Analysis of relative gene expression data using real-time quantitative PCR and the 2(-Delta Delta C(T)) method. Methods 25: 402-408, 2001

24. Zhou W, Wang J, Man WY, Zhang QW and Xu WG: siRNA silencing EZH2 reverses cisplatin-resistance of human non-small cell lung and gastric cancer cells. Asian Pac J Cancer Prev 16 2425-2430, 2015.

25. Jalava SE, Urbanucci A, Latonen L, Waltering KK, Sahu B, Jänne OA, Seppälä J, Lähdesmäki H, Tammela TL and Visakorpi T: Androgen-regulated miR-32 targets BTG2 and is overexpressed in castration-resistant prostate cancer. Oncogene 31: 4460-4471, 2012.

26. Small EJ, Carducci MA, Burke JM, Rodriguez R, Fong L, van Ummersen L, Yu DC, Aimi J, Ando D, Working P, et al: A phase I trial of intravenous CG7870, a replication-selective, prostate-specific antigen-targeted oncolytic adenovirus, for the treatment of hormone-refractory, metastatic prostate cancer. Mol Ther 14: 107-117, 2006.

27. Pol J, Buqué A, Aranda F, Bloy N, Cremer I, Eggermont A, Erbs P, Fucikova J, Galon J, Limacher JM, et al: Trial watch-oncolytic viruses and cancer therapy. Oncoimmunology 5: e1117740, 2016

28. de Gruijl TD, Janssen AB and van Beusechem VW: Arming oncolytic viruses to leverage antitumor immunity. Expert Opin Biol Ther 15: 959-971, 2015.

29. Twumasi-Boateng K, Pettigrew JL, Kwok YYE, Bell JC and Nelson BH: Oncolytic viruses as engineering platforms for combination immunotherapy. Nat Rev Cancer 18: 419-432, 2018.

30. Delwar Z, Zhang K, Rennie PS and Jia W: Oncolytic virotherapy for urological cancers. Nat Rev Urol 13: 334-352, 2016.

31. Fang W, Ping WH, Fang CX, Yuan LC and Qian H: Construction and characterization of oncolytic adenovirus controlled under heat shock protein70 gene promoter. Prog Biochem Biophys 2009: 1536-1543, 2009.

32. Li J, You Y, Yue W, Yu H, Lu T, Wu Z, Jia M, Ruan Y, Liu J, Zhang D and Wang L: Chromatin remodeling gene EZH2 involved in the genetic etiology of autism in Chinese Han population. Neurosci Lett 610: 182-186, 2016.

33. Simon JA and Lange CA: Roles of the EZH2 histone methyltransferase in cancer epigenetics. Mutat Res 647: 21-29, 2008.

34. Liu X, Wu Q and Li L: Functional and therapeutic significance of EZH2 in urological cancers. Oncotarget 8: 38044-38055, 2017.
35. Saramaki OR, Tammela TL, Martikainen PM, Vessella RL and Visakorpi T: The gene for polycomb group protein enhancer of zeste homolog 2 (EZH2) is amplified in late-stage prostate cancer. Genes Chromosomes Cancer 45: 639-645, 2006.

36. van Leenders GJ, Dukers D, Hessels D, van den Kieboom SW, Hulsbergen CA, Witjes JA, Otte AP, Meijer CJ and Raaphorst FM: Polycomb-group oncogenes EZH2, BMI1, and RING1 are overexpressed in prostate cancer with adverse pathologic and clinical features. Eur Urol 52: 455-463, 2007.

37. Bryant RJ, Cross NA, Eaton CL, Hamdy FC and Cunliffe VT: $\mathrm{EZH} 2$ promotes proliferation and invasiveness of prostate cancer cells. Prostate 67: 547-556, 2007.

38. Cai H, Memarzadeh S, Stoyanova T, Beharry Z, Kraft AS and Witte ON: Collaboration of Kras and Androgen receptor signaling stimulates EZH2 expression and tumor propagating cells in prostate cancer. Cancer Res 72: 4672-4681, 2012.

39. Lu C, Han HD, Mangala LS, Ali-Fehmi R, Newton CS, Ozbun L, Armaiz-Pena GN, Hu W, Stone RL, Munkarah A, et al: Regulation of tumor angiogenesis by EZH2. Cancer Cell 18: 185-197, 2010.

40. Xu ZQ, Zhang L, Gao BS, Wan YG, Zhang XH, Chen B, Wang YT, Sun N and Fu YW: EZH2 promotes tumor progression by increasing VEGF expression in clear cell renal cell carcinoma. Clin Transl Oncol 17: 41-49, 2015.

41. Wu C, Jin X, Yang J, Yang Y, He Y, Ding L, Pan Y, Chen S, Jiang $\mathrm{J}$ and Huang $\mathrm{H}$ : Inhibition of EZH2 by chemo- and radiotherapy agents and small molecule inhibitors induces cell death in castration-resistant prostate cancer. Oncotarget 7: 3440-3452, 2016.

42. Debeb BG, Gong Y, Atkinson RL, Sneige N, Huo L, GonzalezAngulo AM, Hung MC, Valero V, Ueno NT and Woodward WA: EZH2 expression correlates with locoregional recurrence after radiation in inflammatory breast cancer. J Exp Clin Cancer Res 33: 58, 2014

43. Trujillo KA, Hines WC, Vargas KM, Jones AC, Joste NE, Bisoffi $\mathrm{M}$ and Griffith JK: Breast field cancerization: Isolation and comparison of telomerase-expressing cells in tumor and tumor adjacent, histologically normal breast tissue. Mol Cancer Res 9: 1209-1221, 2011.

44. Komata T, Kanzawa T, Kondo Y and Kondo S: Telomerase as a therapeutic target for malignant gliomas. Oncogene 21: 656-663, 2002.

45. Köchling M, Ewelt C, Fürtjes G, Peetz-Dienhart S, Koos B, Hasselblatt M, Paulus W, Stummer W and Brokinkel B: hTERT promoter methylation in pituitary adenomas. Brain Tumor Pathol 33: 27-34, 2016

46. Tang J, Wang Z, Li X, Li J and Shi H: Human telomerase reverse transcriptase expression correlates with vascular endothelial growth factor-promoted tumor cell proliferation in prostate cancer. Artif Cells Blood Substit Immobil Biotechnol 36: 83-93, 2008.

47. March-Villalba JA, Martínez-Jabaloyas JM, Herrero MJ, Santamaria J, Aliño SF and Dasí F: Cell-free circulating plasma hTERT mRNA is a useful marker for prostate cancer diagnosis and is associated with poor prognosis tumor characteristics. PLoS One 7: e43470, 2012.

48. Zhou J, Wang H, Cannon V, Wolcott KM, Song H and Yates C: Side population rather than $\mathrm{CD} 133^{+}$cells distinguishes enriched tumorigenicity in hTERT-immortalized primary prostate cancer cells. Mol Cancer 10: 112, 2011.

49. Abdelrahman AE, Arafa SA and Ahmed RA: Prognostic value of Twist-1, E-cadherin and EZH2 in prostate cancer: An immunohistochemical study. Turk Patoloji Derg 1: 198-210, 2017.

50. Beavon IR: The E-cadherin-catenin complex in tumour metastasis: Structure, function and regulation. Eur J Cancer 36: $1607-1620,2000$

51. Harrington KJ: The biology of cancer. Medicine 44: 1-5, 2016.

52. Bullwinkel J, Baron-Lühr B, Lüdemann A, Wohlenberg C, Gerdes J and Scholzen T: Ki-67 protein is associated with ribosomal RNA transcription in quiescent and proliferating cells. J Cell Physiol 206: 624-635, 2006.

53. Yoo KH and Hennighausen L: EZH2 methyltransferase and H3K27 methylation in breast cancer. Int J Biol Sci 8: 59-65, 2012. 